\title{
Research
}

Nathan Hodson, Elizabeth Ford and Maxwell Cooper

\section{Adherence to guidelines on documentation required for registration to London GP practice websites:}

\author{
a mixed-methods cross-sectional study
}

\begin{abstract}
Background

The most common obstacle to registration with a GP practice in the UK is difficulty presenting proof of address. NHS guidelines stipulate that inability to provide ID or proof of address is not reasonable grounds to refuse registration. Practices may ask patients to present ID/proof of address, but need a policy in case patients cannot.
\end{abstract}

Aim

To find out how many London GP practice websites ask for documentation without a policy for where this cannot be provided and compare how GP practice websites describe the registration process in patient-facing material.

\section{Design and setting}

Cross-sectional study of practices from 10 London boroughs $(n=100)$.

\section{Method}

A proforma was piloted and then implemented, recording whether practices 'demanded', 'requested', or 'mentioned' photo ID or proof of address and whether there was a plan for patients without documentation. Text relating to documentation from all 100 practices for registration was subjected to thematic analysis.

\section{Results}

Out of 100 practices $75 \%$ asked for

documentation. The majority of these were

'demanded'. A plan was included for people without documentation in $12 \%$ of practice websites. Five themes emerged from analysis of website content: reassuring people without documentation; diverse requirements

between practices; conflating administration and treatment; withholding treatment; and immigration and ethnicity.

\section{Conclusion}

Many practice websites breached NHS Standard Operating Principles and possibly the Equalities Act 2010. All practices should create a clear policy for patients who do not have photo ID/ proof of address (for example, including a named receptionist), and update their websites accordingly.

\section{Keywords}

equal rights; general practice websites; health services accessibility; human rights; primary care.

\section{INTRODUCTION}

Accessibility is central to the mission of primary care in the UK. The NHS Standard Operating Principles in England state inability to provide identification or proof of address is not reasonable grounds to refuse registration.

Primary care is mainly provided by GPs in the UK. Despite this core value of accessibility, $17 \%$ of Londoners are not registered with a GP and the most common obstacle to registration is presenting proof of address. ${ }^{2}$ People who are not registered with a GP miss out on health benefits of primary care, increasing strain on the wider health system; in 2014 over 150000 patients without a GP attended emergency departments in London hospitals.

Some groups of people are at greater risk of exclusion from primary care than others, particularly homeless people, Travellers, sex workers, and drug users, as well as recent arrivals to the UK. ${ }^{4}$ Members of these groups report finding it particularly difficult to present the right documents at a GP practice for registration. ${ }^{4}$ Charitable organisations such as Homeless Link and Healthwatch report that many patients have been denied registration at GP practices because of their inability to present the correct documentation. 2,5,6 Citizens Advice research found that $58 \%$ of GP surgeries refused to register patients without specific documentation.? Not only are members of excluded groups less likely to have

N Hodson, BSc, BMBS, DHMSA, student (MPH) previously at University Hospitals of Leicester, University of Leicester, College of Medicine, Biological Sciences and Psychology, Leicester. E Ford, MA, DPhil, senior lecturer in primary care research, Department of Primary Care and Public Health; M Cooper, PhD, MPC, MRCGP, DRCOG, DTMH, senior lecturer in primary care, Brighton and Sussex Medical School, Division of Primary Medicine, Brighton stable accommodation land therefore proof of address) but they are also more likely to struggle with :... poor literacy and the subsequent inability to complete applications ...' and are strongly influenced by small financial penalties. ${ }^{4}$ This presents a barrier to completing GP registration forms as well as paperwork for a passport or driver's licence, and makes the cost of these documents ( $€ 75.50$ and $€ 34.00$ ) a significant disincentive. ${ }^{8,9}$ Statistics from the Office for National Statistics (ONS) and the Driver and Vehicle Licensing Agency (DVLA) show that $17 \%$ of British adults do not have a passport and 30\% lack a driving licence..$^{10,11}$

Taken together, these factors suggest some vulnerable groups may be highly influenced in their ability to register with a GP by the information available about practices rules on registration documentation. GPs often give such information on their publicfacing websites, but struggle to keep this information up to date. Practice websites and NHS Choices pages have been shown to include spurious information about staff and opening hours. ${ }^{12,13}$ Local Healthwatch activists have drawn attention to the 19 out of 42 Greenwich GP practice websites that imply documentation is a prerequisite for registration. ${ }^{14}$ Studying GP practice websites may be an unreliable way of discovering practices true policy, but is the only information that new registrants are likely to access, short of visiting the clinic in person.

\section{Address for correspondence}

Nathan Hodson, Harvard TH Chan School of Public Health, 677 Huntington Ave, Boston, MA 02115, US. Email: nh227ale.ac.uk

Submitted: 6 February 2019; Editor's response: 3 April 2019; final acceptance: 9 May 2019. @British Journal of General Practice This is the full-length article (published online 24 Sep 2019) of an abridged version published in print. Cite this version as: Br J Gen Pract 2019; DOI: https://doi.org/10.3399/bjgp19X705581 


\begin{abstract}
How this fits in
Many patients have reported that, contrary to NHS Standard Operating Principles and BMA guidelines, their inability to provide documentation meant they were denied registration at a GP practice. GP practice websites are an important but often unreliable source of information about registration. This study found that $75 \%$ of London GP practice websites state that documentation will be involved in the registration process as well as conflating administrative tasks with treatment - asserting that urgent treatment is documentation dependent - and misinterpreting regulations on immigration status. The authors demonstrate heterogeneity between policies, which implies that the most stringent policies are excessive, and identify examples of pragmatic good practice (12\%) where GP practice websites clearly include a plan for those who cannot provide documentation.
\end{abstract}

The way that practices advertise services should be guided by Good Medical Practice:

When advertising your services, you must make sure the information you publish is factual ... ${ }^{15}$

Thus, although the GP practice may operate a different policy than it advertises online (which would breach Good Medical Practice guidance), the claim that documentation is required for registration could deter people from trying to register,

\section{Box 1. Summary and quotes from NHS London guidelines on patient registration ${ }^{16}$}

The practice must take steps to ensure a patient receives care and should not withhold treatment from patients who need it

neither registration or appointments to see the doctor should be delayed because of the unavailability of a new patient check appointment.

'Registration and appointments should not be withheld because a patient does not have the necessary proof of residence or personal identification.

General practices are also under a duty to provide emergency or immediately necessary treatment where clinically necessary irrespective of nationality or immigration status. They are also required to provide 14 days of further cover following provision of immediate and necessary treatment.

\section{Immigration status is irrelevant so practices should not even ask about it}

Immigration status does not affect eligibility to primary care (see page 7 [of Guidelines ${ }^{16}$ ] for secondary care regulations) - practices should not enquire about patients' immigration status.

'There is no set length of time that a patient must reside in the UK in order to become eligible to receive NHS primary care services.

A patient does not need to be "ordinarily resident" in the UK to be eligible for NHS primary care - this only applies to secondary care

'The length of time that a patient is intending to reside in an area in the UK [dictates] whether a patient ... is registered as a temporary or permanent patient. even if they might encounter a more hospitable approach should they attend the surgery.

\section{Law and guidelines}

According to NHS Standard Operating Principles, inability to present proof of address or photo ID is not acceptable grounds to deny a patient registration at a GP practice. ${ }^{1}$ This is a reference to the NHS GP contract section 13.7.1, which states:

'The contractor may only refuse an application ... if the contractor has reasonable grounds for doing so which do not relate to the applicant's age, appearance, disability or medical condition, gender or gender reassignment, marriage or civil partnership, pregnancy or maternity, race, religion or belief, sexual orientation or social class.'17

The British Medical Association (BMA) reiterates the NHS Standard Operating Principles:

'There is no contractual duty to seek evidence of identity or immigration status or proof of address. Therefore practices should not refuse registration on the grounds that a patient is unable to produce such evidence. 18

It is reasonable for practices to decline patients on the basis that they live out of area, but not to refuse care to those who cannot provide proof of address. ${ }^{1}$ In addition, the Standard Operating Principles recognise that it may be useful for a practice to ask to see ID, but must specify: what action should be taken when a patient is unable to supply any form of ID. " If a patient states that they live in the area then no other proof is needed.

The NHS London guidelines on registration go even further and are summarised in Box 1.16

The Equality Act 2010 applies to GP practices and is breached by policies that treat people differently or worse because of a protected characteristic such as race. This is summed up in the Standard Operating Principles:

Any practice policy to ask for patient ID should be applied in a non-discriminatory fashion."

Given that GP practice websites are the only available public-facing source of information about the GP practice's registration rules for new patients, these are 


\section{Box 2. Data collection proforma}

1. Whether or not the website mentioned documents required for registration.

2. Whether the documentation required was photo ID, or proof of address, or both.

3. Whether documentation was: - necessary for registration ('you must bring'); - requested politely ('please bring'); or - mentioned in passing ('if you have').

4. Whether any alternative was described in the case that such documents were not held by the registrant (reassurance, flexibility).

a useful proxy for understanding if practices are adhering to national policies in a nondiscriminatory way. GP practice websites in London were therefore examined, with the aim of quantifying how often these sites breached guidelines on registration documentation requirements, specifically, how many sites required ID and/or proof of address for registration and failed to offer an alternative policy. This study explored how this was explained in the registration information on GP practice websites.

\section{METHOD}

\section{Study design}

A cross-sectional analysis of the websites of GP practices in London was undertaken. Both a quantitative approach and a qualitative approach were used.

\section{Selection of GP practices}

Ten London boroughs were selected at random. The names of all GP practices in 10 London boroughs were obtained from clinical commissioning groups. A random number generator (https://www.random.org/) was used to select 10 practices from each of these boroughs to generate a representative sample totalling 100 GP surgeries $17.5 \%$ of all surgeries in London). Two stages of random sampling were used to ensure a diverse sample from across London without giving preference to communities with high levels of deprivation, as every borough in London has areas of deprivation. Surgeries were excluded if they focused on alternative medicine, catered to a particular group (for example, people who were homeless), and/ or had no website. The random number generator was used to select another practice from the same borough.

\section{Data collection}

Piloting and development of a proforma for practice data collection (Box 2) took place during July 2017, which was constructed in
Microsoft Excel. GP practice websites were accessed between 9 October 2017 and 17 October 2017.

Where there was uncertainty in strength of wording on the practice website, the practice was scored as the less strongly worded option.

In addition to the structured data on the proforma, all text relating to registration and documentation was extracted from the website, to be analysed qualitatively.

\section{Data analysis}

Structured data were summarised descriptively, because of small numbers.

Qualitative data were analysed using a thematic analysis according to the six stages outlined by Braun and Clarke. ${ }^{19}$ Initial coding of the data was conducted. Themes were developed inductively, tested for internal homogeneity and external heterogeneity following Patton, ${ }^{20}$ and key quotations were selected for inclusion.

For anonymity, practice names are not included in the manuscript; only the borough and a designated practice number is shown.

\section{RESULTS}

\section{Quantitative analysis}

After 12 exclusions from the 112 practices looked at, 100 surgeries were included. Of these 100 practices, $75 \%$ asked for documentation. Sixty-five practice websites mentioned that photo ID was required for registration, and 72 required proof of address. Sixty-two practices mentioned both photo ID and proof of address, and 25 did not mention either (Figure 1).

Among the 65 that mentioned photo ID, 33 of these $(51 \%)$ used language such as 'required for registration', 21 (32\%) made a request ('please bring'), and 11 (17\%) mentioned documentation in passing ('it would help if you could bring') without a specific request. Of the 72 that mentioned proof of address, 39 (54\%) required proof of address, 26 (36\%) requested, and 7 (10\%) mentioned it in passing.

Only 12 of the 75 websites that mentioned either photo ID or proof of address included a comment suggesting that it might be possible to obtain care without this documentation.

Figure 2 shows how many practice websites reported a policy for patients without documentation.

\section{Thematic analysis}

Thematic analysis generated five themes within the language used to describe requirement of documentation for 


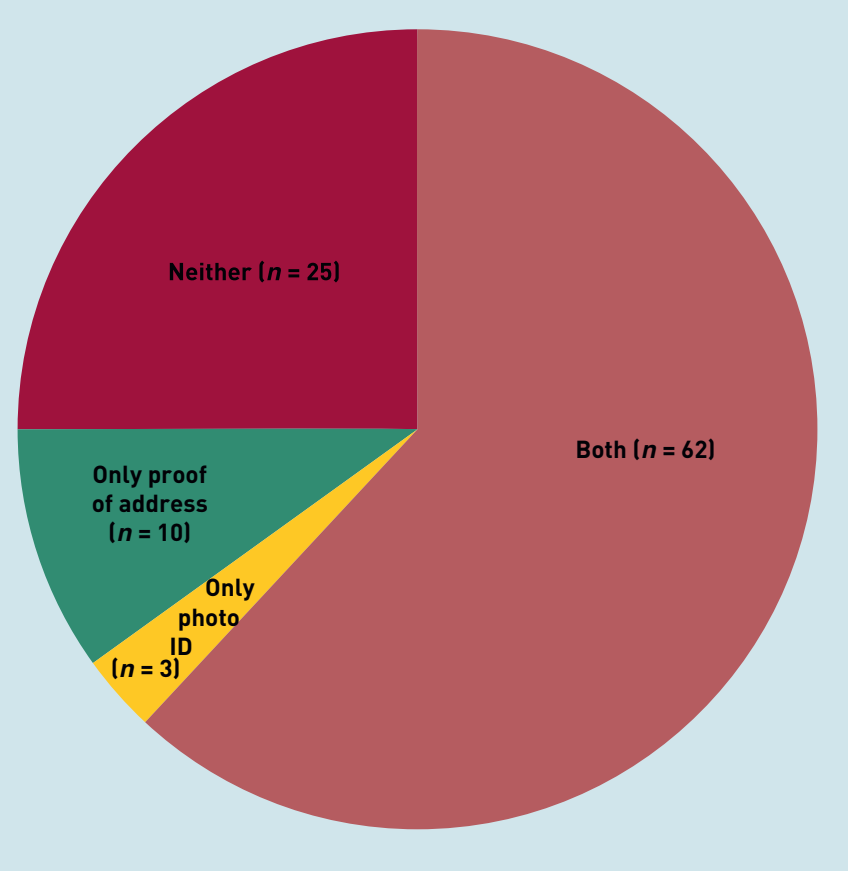

Figure 1. Proportion of documentation mentioned on GP practice websites.

Figure 2. Proportion of practice websites reporting a policy for patients without documentation. registration, four representing failure to follow guidance, and one indicating positive language and policies that matched Standard Operating Principles and guidelines.

Heterogeneous demands. There was diversity between documents required by practices. Although most websites suggested that documentation was involved

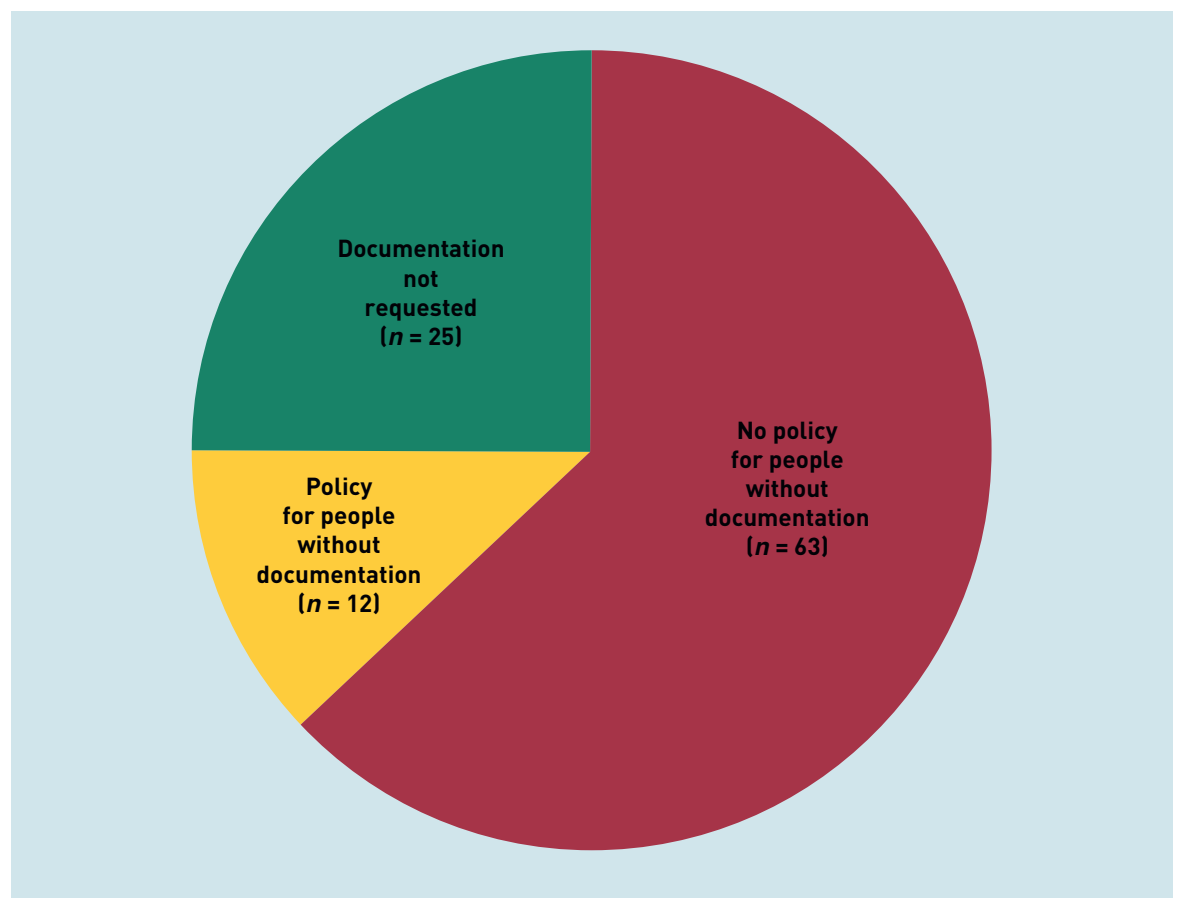

in registration, only one practice website correctly stated that this was not the case:

'To register and receive treatment at a GP practice: You do not need a fixed address; You do not need identification; Your immigration status does not matter.' (Kensington and Chelsea 9)

There was also heterogeneity among practices that required documents. Some accepted tenancy agreements, while others did not.

We do not accept: tenancy agreements/ job centre letters/payslips as a proof of address.' (Newham 9)

Some accepted mobile phone bills (Kensington and Chelsea 6), while many others specifically stated that they did not (for example, Camden 8, Newham 8, Haringey 10). One practice went even further, apparently declining landline bills:

We do not accept telephone or bank statements. '(Richmond-upon-Thames 2)

Most practices asked for a driving licence, although some stated that it could not be used as proof of address (Camden 3), or could be used as either proof of address or photo ID, but not both (Kensington and Chelsea 6). One website specified that a provisional licence was acceptable (Wandsworth 9), but another stated the opposite:

Learners Driving Licence will not be accepted. '(Camden 4)

Two practices accepted student ID, either from University College London (Camden 9) or King's College London (Westminster 3). Other practices did not mention student cards.

The duration of bills also varied. Many practices asked for a bill from the last 3 months. One website was more stringent, demanding a bill from the last 2 months (Camden 10). Inconsistency was evident on another page that sought proof of address from '... the last 3 months' and '... an official notification dated within the last calendar month' (Richmond-upon-Thames 4).

This practice is also unusual in requesting consecutive bills, rather than a single recent bill:

Last 3 months of bills: e.g. gas/telephone/ electric OR e.g. 3 months of bank statements.' (Richmond-upon-Thames 4) 
Most practices considered a single bill sufficient proof of address. Demanding serial bills over several months presents a significant impediment to anybody new to the area. Other practice websites asked new patients to provide two bills in addition to photo ID (Kensington and Chelsea 10 and Haringey 9), although it seems these could be different bills rather than the consecutive copies of the same bill.

Some websites described policies that are clearly unenforceable:

All patients aged 16 years and over are requested to provide photographic proof of identity (e.g. passport, photocard driving license [sic]) and current proof of address (e.g. utility bill no older than 3 months). (Haringey 4)

Reassuring comments. Several practices already acknowledged that their website could create a barrier to registration for certain patients without documents. One practice recognised that new registrants may not have a medical card, phrasing their request carefully or encouraging patients without the preferred paperwork to speak with receptionists:

'Please note that we are always open to discuss this matter as we are aware that some individuals may have problems providing the requested ID.' (Richmond upon Thames 2)

One practice even named the receptionist who could be spoken to:

Where such a letter is not available, we would encourage the prospective patient to talk to our Lead Receptionist [name] who can facilitate registration.' (Haringey 10)

Two neighbouring practices used identical text, which included the phrase:

If you can not [sic] provide these documents immediately we'll register you temporarily (for 3 months) so that you have time to obtain them.' (Tower Hamlets 2 and Tower Hamlets 4).

Another practice website had copied and pasted the NHS London guidance (Richmond-upon-Thames 7), demonstrating awareness of the problem, although it is not easily interpretable to the layperson.

Conflating administration and treatment. In some practices the distinction between registration and care became blurred.
Practice websites claimed that registration was contingent on the provision of medical documents such as a vaccination history Ifor example, Newham 2 and Westminster 8) or even attending a health check:

'You will be required to book for a health check as part of the acceptance procedure. (Newham 7)

You will need to attend the surgery within 30 days to sign the registration and consent forms and to have your blood pressure checked. '(Southwark 2)

One practice website implies a state of partial or incomplete registration prior to a health check, although it is not clear whether this involves withholding treatment:

We are unable to register you fully until this has been done.' (Richmond-uponThames 8)

Others simply encouraged attendance at the health check:

An appointment will be offered with our Health Care Support Worker during your registration.' (Southwark 3)

Patients were asked to perform some administrative tasks that could easily be performed by the surgery. Two practice websites asked people applying for registration to photocopy their documents before attending (Richmond-upon-Thames 10 and Ealing 10). Others implied that registration depended on patients finding out their NHS number from their last practice (for example, Wandsworth 7).

One website reported restrictive timings for registration:

If you would like to register please come to the surgery between 10:30 a.m. and 11:30 a.m., Monday to Friday. '(Newham 5)

The administrative task of getting the preferred documentation seems to supersede the aim of providing treatment according to some practice websites.

Withholding treatment. Contrary to the guidelines, some practice websites suggest that emergency treatment will not be provided until documents are provided:

Until you have submitted your ID you will not be registered at the practice and cannot be seen for any treatment.' (Kensington and Chelsea 1) 
One practice explicitly contradicts guidelines by stating that failure to submit ID will delay registration, when patients should instead be registered first and asked for ID at a later date as required:

Failure to provide these will delay your registration with this surgery.' (Haringey 5)

Another practice website minimises the practice's responsibility to provide emergency treatment, including people in the process of registering, by offering an alternative:

If you are very unwell and have no GP you can visit a walk-in centre whilst awaiting registration e.g. [address and phone number given] ... Until you have submitted your ID you will not be registered at the practice. (Westminster 4)

Immigration and ethnicity. Two GP practice websites openly admitted to treating British citizens differently from non-British people:

If you have come from abroad you will need to show us your passport, ID card or Home Office correspondence.' (Haringey 6)

If you have recently arrived in the country, [you will need to bring in] details of your visa entry permissions.' (Southwark 9)

If it was practice policy to require different documents from people based on ethnicity or some undefined notion of having 'recently arrived' then this could fail to comply with the Equality Act 2010.

One practice misused the term "normally resident', which only applies to secondary care (Haringey 4), and breached NHS London guidelines by making specific demands relating to immigration status:

Immigration Status - We require one of the following: An up-to-date passport OR If you are not normally resident in the UK, a letter from the immigration department stating how long you have been permitted to stay in the country OR If you are not normally resident in UK an ID card from the immigration department.' (Haringey 4)

Another practice describes patients being 'registered with the NHS' or having 'entitlement to NHS'. These are neither formal concepts nor features of the guidelines (Camden 4). This website goes on to use capital letters and potentially menacing language to make stringent demands of people who are new to the UK, suggesting that whoever wrote the website completely misunderstands who is eligible for treatment:

If you have never been registered with the NHS before, you will need to provide us with: The exact date, month and year of your entry to the UKPROOFOFENTITLEMENT TONHS (NATIONAL HEALTH SERVICE) TREATMENT In order to establish patient's entitlement to NHS ... Any incorrect information may result in the rejection of your registration from Patient Data Department.' (Camden 4)

Some practice websites implied registration depended on nationality by mentioning an 'Ethnicity form (Wandsworth 4) which could be off-putting. although it may well refer to a form for collecting equality data. Other practices use vague language allowing receptionists wide discretion:

If requested be able to provide the following: [ID] ... You may be asked to bring in proof of identity.' (Westminster 10)

'[Reception staff] will explain the necessary procedures.' (Wandsworth 8)

One practice website denies singling out certain people, implying an understanding of the law, but nevertheless mistakenly suggests that people who are new to the country are ineligible to register with a GP:

We need to know that you have been resident in the UK for at least the last 3 months. This is our Practice Policy for ALL patients wishing to register with us.' (Richmond-upon-Thames 4)

\section{DISCUSSION}

\section{Summary}

The authors aimed to quantify how often websites of London GP practices breached guidelines on registration documentation requirements and to explore what websites said about registration. A wide disparity was found in documents required for patient registration between websites. Many examples conflicted with NHS policy. The majority of GP practice websites (75 out of 100) stated that providing documentation was part of the registration process. Only $12(16 \%)$ of these reassured those without documentation that receptionists could help. In the qualitative analysis of website content it was found that practices made heterogeneous requirements for documents, conflated administrative processes of registration with access to 
treatment, which may lead to treatment being withheld, and misinterpreted regulations about immigration status or nationality. Examples of good practice were also found where GP practices showed flexibility and openness to registrants who could not provide documentation.

\section{Strengths and limitations}

This study is novel in exploring online barriers to GP access, in particular whether guidelines are in fact being broken. The study benefits from the clear guidelines (and law) against which information on GP practice websites can be tested and has a large sample of practices. Another strength of this article is that it undertakes an in-depth textual analysis allowing for identification of good areas of practice.

Although the General Medical Council demands that GP websites are accurate, it is possible that they do not represent actual policy. Likewise, patient perspectives and behaviours have not been investigated. Two of the authors practise, or have practised, in primary care; this means their analysis of this data has been informed by a positive, but not rose-tinted, view of staff working in GP practices. No practice staff were interviewed in this analysis. As such the reasoning and motivations of staff who write these policies could not be elaborated from this source of data.

All practices sampled were in London. General practices in other areas in the UK may show different policies because of less transient populations than London; however, local Healthwatch groups have found corroborating stories around the UK. ${ }^{14}$

\section{Comparison with existing literature}

The quantitative results support the findings of informal local surveys of GP websites by Healthwatch groups elsewhere in the country, which have suggested that these findings may not be confined to London. ${ }^{14-21}$ One limitation of such surveys is that they did not show whether documentation was mentioned in passing (not necessarily a breach of guidelines) or demanded (a definite breach of guidelines). This article shows that most practice websites mentioning documentation implied it was essential for registration.

Diverse policies were found. Most practices stated that ID and proof of address were required, offering no alternative for people without ID, thereby breaching NHS England guidelines.' Refusal to accept tenancy agreements and mobile phone bills presents a particular barrier to people who often move address, who are also those most likely to need to register at a new practice frequently. One practice requested 3 months of consecutive bills, which could preclude registration until patients have spent 4 months in the area, a particular problem for people with chronic diseases who need regular check-ups.

For conflating administration and treatment, in direct contravention of NHS England guidelines, evidence was found that some practices threatened to withhold treatment until patients provided ID or attended a health check, that patients could only register during 1 hour in late morning, and that patients could not be seen for emergency treatment. ${ }^{1}$ These restrictions on treatment may endanger patients and divert them into the more expensive emergency department.

The alarming suggestion that patients could not be seen for emergency treatment contradicts NHS England guidelines:

General Practices are also under a duty to provide emergency or immediately necessary treatment, where clinically necessary, irrespective of nationality or immigration status. The practice is required to provide 14 days of further cover following provision of immediate and necessary treatment."

The cost-effective GP system ensures that $A \& E$ is reserved for people requiring expensive hospital care; preventing people from accessing primary care has expensive knock-on effects. ${ }^{3}$ Primary care is under pressure too, potentially suggesting that practices implement these barriers to reduce the flow of work. Administration teams within GP practices are increasingly praised for coming up with ways of reducing the doctors' workload, 22,23 but initiatives such as asking patients to photocopy their own documents are likely to delay registration for some patients.

For immigration and ethnicity, the authors demonstrated that the information on some GP websites was legally questionable under the Equality Act 2010. Furthermore, the Health and Social Care Act 2012 makes all NHS bodies responsible for tackling inequality. Other practices risked facing accusations of discrimination because they lacked a firm policy but demanded documentation at the receptionists' discretion. Some misused or invented concepts indirectly discriminating against people from other countries without experience of the NHS. This is despite guidance that immigration status is of no relevance to GP registration. ${ }^{17}$. 
Problems with race and migration may run deeper within the NHS. Racial inequality in the health service is welldocumented. ${ }^{24,25}$ The NHS Staff Survey 2015 found that, compared with white staff, black and minority ethnic (BME) staff report equal levels of harassment from the public; however, BME staff face higher levels of harassment from colleagues. ${ }^{26}$ This study builds on knowledge that there is more work to do to improve NHS staff's approach to racial diversity, revealing that in some instances policies are wrongly impairing access to care.

\section{Implications for research and practice}

This article has not only revealed a significant diversity in policy, but has also uncovered examples of good practice relating to both local policies and website instructions.
In the long term, GP practices may all operate their online presence from a central platform such as NHS Choices rather than disparate independent efforts. ${ }^{13}$ One solution to the heterogeneity discovered here would be for all GP practice web pages to display a set and approved statement about rights to access, taken from NHS guidelines, and trialled for language and acceptability with members of the public.

This would make it easier to ensure conformity between practice policies and could lead to a single national policy. Future research should question staff in GP practices on why these disparities arise. In the UK, everybody has the right to access primary care and it is essential to ensure that neither GP practice policy, nor erroneous online information, deter patients from accessing primary health care.

\section{Funding}

This research was unfunded.

\section{Ethical approval}

Ethical approval was not sought as all websites were in the public domain.

\section{Competing interests}

The authors have declared no competing interests.

\section{Discuss this article}

Contribute and read comments about this article: bjgp.org/letters 


\section{REFERENCES}

1. NHS England. Patient registration standard operating principles for primary medical care (general practice). 2015. https://www.england.nhs.uk/south/ wp-content/uploads/sites/6/2018/01/patient-registrations.pdf laccessed 11 Sep 2019)

2. Healthwatch News. Do you need proof of address to register with a GP? 2016. https://www.healthwatch.co.uk/news/2016-01-08/do-you-need-proof-addressregister-gp (accessed 11 Sep 2019).

3. Savage, J. More than 150,000 A\&E patients in London 'not GP-registered'. BBC News 2015; 12 Nov: https://uww.bbc.co.uk/news/uk-england-london-34789785 laccessed 11 Sep 2019).

4. O'Donnell P, Tierney E, O'Carroll A, et al. Exploring levers and barriers to accessing primary care for marginalised groups and identifying their priorities for primary care provision: a participatory learning and action research study. Int J Equity Health 2016; 15(1): 197.

5. Sparrow N. Nigel's surgery 29: Looking after homeless patients in general practice. 2017. https://uww.cqc.org.uk/guidance-providers/gps/nigels-surgery29-looking-after-homeless-patients-general-practice laccessed 11 Sep 2019).

6. Homeless Link. Health needs audit - explore the data. https://www.homeless. org.uk/facts/homelessness-in-numbers/health-needs-audit-explore-data laccessed 11 Sep 2019).

7. Citizens Advice. Registering frustration. Could a simpler, clearer GP registration process help manage demand in the health system and improve the patient experience?2015. https://www.citizensadvice.org.uk/Global/CitizensAdvice/ Public\%20services\%20publications/Registering_frustration_Citizens_Advice.pdf laccessed 11 Sep 2019).

8. GOV.UK. Renew or replace your adult passport. 2019. https://www.gov.uk/ renew-adult-passport laccessed 11 Sep 2019).

9. GOV.UK. Driving licence fees. 2019. https://mww.gov.uk/driving-licence-fees laccessed 11 Sep 2019).

10. Office for National Statistics. Detailed country of birth and nationality analysis from the 2011 Census of England and Wales. 2013. https://webarchive. nationalarchives.gov.uk/20160107124139/http://www.ons.gov.uk/ons/ dcp171776_310441.pdf (accessed 11 Sep 2019).

11. Driver and Vehicle Licensing Agency. Freedom of information request. 2015. https://assets.publishing.service.gov.uk/government/uploads/system/uploads/ attachment_data/file/397430/FOIR4341_How_many_people_hold_licences_in_ the_UK.pdf laccessed 11 Sep 2019).

12. Anderson $\mathrm{H}$, Adamson J, Birks Y. Presenting the primary care team to the public: a qualitative exploration of general practice websites. Br J Gen Pract 2018; DOI: https://doi.org/10.3399/bjgp18X695009.
13. Richards EC, Cowling TE, Gunning EJ, et al. Online data on opening hours of general practices in England: a comparison with telephone survey data. $\mathrm{Br} \mathrm{J}$ Gen Pract 2015; DOI: https://doi.org/10.3399/bjgp15X687841.

14. Healthwatch Greenwich. Accessing GP services in Greenwich. 2018. https:// healthwatchgreenwich.co.uk/wp-content/uploads/2018/04/GP-Access-ReportJune-18.pdf laccessed 11 Sep 2019).

15. General Medical Council. Good medical practice. Domain 4: maintaining trust. 2013. https://www.gmc-uk.org/ethical-guidance/ethical-guidance-for-doctors/ good-medical-practice/domain-4---maintaining-trust laccessed 11 Sep 2019).

16. NHS London. GP patient registration. 'Once for London' pan-London operating principles for primary care. https://www.lmc.org.uk/visageimages/ news/2012/150812LondonGPPatientRegistrationsOperatingProcedures.pdf laccessed 11 Sep 2019).

17. NHS England. Standard general medical services contract 2017/18. 2018. https://www.england.nhs.uk/wp-content/uploads/2018/01/17-18-gms-contract. pdf (accessed 11 Sep 2019).

18. British Medical Association. Patient registration for GP practices. 2018. https:// unw.bma.org.uk/advice/employment/gp-practices/service-provision/patientregistration-for-gp-practices (accessed 11 Sep 2019).

19. Braun V. Clarke V. Using thematic analysis in psychology. Qual Res Psychology 2006, 3(2): 77-101.

20. Patton MQ. Qualitative evaluation and research methods. 2nd edn. Newbury Park, CA: Sage, 1990.

21. Healthwatch Dorset. Registering with a GP practice and booking appointments. 2017. https://www.patientlibrary.net/cgi-bin/downloadhw. cgi?file=158733; gen=d3pX1h5hplJV8681BMIMA1 gXKZAJ\%57CkVBT2oi pajxBlo0M9VCc\&usg=AOVVaw2uvFOVMEkLvNv6_ghklnnV laccessed 11 Sep 2019).

22. NHS England. GP's admin workload significantly reduced. 2019. https://www. england.nhs.uk/gp/case-studies/gps-admin-workload-significantly-reduced/ laccessed 11 Sep 2019).

23. NHS England. Changes in admin team processes help reduce GP pressures - West4GPs, London. 2018. https://www.england.nhs.uk/publication/changesin-admin-team-processes-help-reduce-gp-pressures-west4gps-london/ laccessed 11 Sep 2019).

24. NHS England. Briefing for NHS boards on the NHS Workforce Race Equality. NHS workforce race equality delivers better care, outcomes and performance. 2015. https://www.england.nhs.uk/wp-content/uploads/2015/10/wres-nhsboard-bulletin.pdf laccessed 11 Sep 2019).

25. Middleton J. Is our beloved NHS a racist institution? Nursing Times 2017 https://www.nursingtimes.net/opinion/is-our-beloved-nhs-a-racistinstitution-23-11-2017/ laccessed 11 Sep 2019).

26. NHS Survey Coordination Centre. NHS staff survey. 2015. http://mwn. nhsstaffsurveys.com/Page/1062/Past-Results/Staff-Survey-2015-Detailedspreadsheets/ (accessed 11 Sep 2019) 